\title{
No conjecture is permanently undecidable in real numbers.
}

\begin{abstract}
:
These days, many people are using Turning machines( computers) to prove or disprove a conjecture because:

i) Even billions of verified numbers cannot prove any conjecture as we cannot reach infinity, but it makes the conjecture more and more valid, as we verify up to higher and higher numbers.

ii) Even one number is enough to disprove! So, many turning machine efforts are to find that one number so that one can disprove.
\end{abstract}

Of late, unfortunately, there is a growing third possibility of declaring it as undecidable. Many people are conveniently using undecidable possibility as a big dustbin, throwing every difficult problem in to it. The dust bin is growing bigger and bigger because many new conjectures are being constructed every day, based on some so called undecidable conjectures. The logic that we have applied may make the turning machine to run for ever, before any conclusion is reached. But (at least in real numbers) there will be definitely another logic which will give a finite trials before concluding yes or no to the problem.

Many such conjectures are about prime numbers. All these can be proved or disproved once we establish a fundamental logic for prime numbers. The aim of this paper is to show that No conjecture is permanently undecidable in real numbers. Also, this logic can be extended to any problem in mathematics.

\section{TheNumber line.}

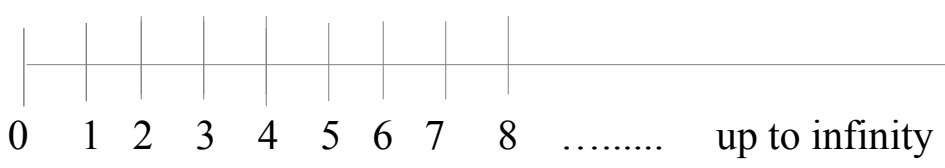

Figure -1. Number line.

Basic logic of the real number line is that adding one to any number $n$ will give $n+1$, the next number. This is because we have defined the (positive) real number line to be like that. So, if we try to verify this with the help of a turning machine, it will be concluded as undecidable, as there are infinite numbers to be done. There is no meaning in saying that we have verified up to several billion numbers and beyond that we do not know.

\section{There is no point in proving our own rule because, if there is a violation anywhere in the number line, it is not the number line that we have defined.}

Again if some one assumes the existence of a natural number neither even nor add, existing in the natural number line, then also the assumption is wrong, as it evident that every number of the form $2 n+1$ must be odd for all natural values of $n$. This is because, even or odd is simply decided by whether the number is divisible by 2 or not. 


\section{Simple examples.}

Let us take the simple statement that

Sum to infinite terms of the series $1+1 / 2+1 / 4+1 / 8+\ldots \ldots=2$.

If we start verifying it using our simple turning machine, then we will be getting $1.99999 . .75$ ( by logic the last digit will be 5 ) for a finite term and it will never give 2, unless we round it off to the previous number. This is happening because of our logic.

Let us do the same thing in a different logic.

Start with the number 2 . After making it $1 / 2$ once, we get $1+1$. let us take the second part, leaving the first part and make it into half of the existing value. It becomes $1+1 / 2+1 / 2$. Doing once again for the last part, we get $1+1 / 2+1 / 4+1 / 4$. We can continue this process infinite times because dividing any small fraction by 2 will never make it zero. The sum is 2 because we started with 2 only and we cannot increase or decrease sum total of all fractions by dividing any number or fraction successively any number of times.

Physically we cannot do and verify the above problem because it requires infinite time. But many people are doing such problems in turning machines, which is nothing but trying to do physically. We have to prove it mathematically with a logic rather than physically doing it using a turning machine.

Can we say that $10 / 3=3.33333=$ undecidable because exact value cannot be written in decimal form? Similarly pi and $\sqrt{2}$, even though the last digit in the decimal form is not possible, we cannot say that exact value is undecidable.

We can call it as incapability of decimal notation or number notation because we can represent these in other forms like exact value of $\mathrm{pi}=$ ratio of circumference of the circle to its diameter.

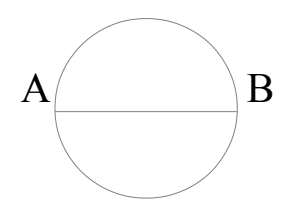

Figure 2. Circle with diameter marked.

Similarly, $\sqrt{2}$ is the length of the side of the square having the area equal to 2 square units.

We can declare undecidable in real numbers only when a portion of the number does not exist or undefined or unpredictable or hidden permanently from the observer. None of the above things can happen to the natural number line, as we know that it extents from 0 to infinity, without any break or irregularity.

\section{Conclusion:}

So, how can we decide that we "cannot decide permanently? "It is actually a matter of time before we decide many of the so called undecidable conjunctures because the turning machine will become smarter and smarter and we can go for a better and better logic with time. In fact, many mathematicians thought Format's last theorem as undecidable for many years and finally it was proved. For any such problem, may get the answer in a year, or 10 years and we know that Time is infinite! 